\title{
Death Profiling of Hospitalized Patients with COVID-19: Experience from a Specialized Hospital in Bangladesh
}

\author{
Md. Shahed-Morshed ${ }^{1}$, Abdullah Al Mosabbir², Mohammad Sorowar Hossain ${ }^{2 *}$ \\ ${ }^{I}$ Emergency medical officer, Kurmitola general hospital, Dhaka, Bangladesh, ${ }^{2}$ Department of Emerging and Neglected Diseases, Biomedical Research Foundation, \\ Dhaka, Bangladesh.
}

\begin{abstract}
The information on characteristics and causes of mortality in deceased patients with coronavirus disease 2019 (COVID-19) is scarce in the literature. This study is aimed to document the clinical profile with causes of death in deceased patients admitted to a COVID-19 dedicated hospital in Dhaka, Bangladesh. This cross-sectional retrospective study included 108 RT-PCR confirmed COVID-19 associated deceased patients admitted in Kurmitola general hospital, Dhaka, Bangladesh, between 25 March 2020 and 24 June 2020) Data were collected from hospital records. Causes of death were categorized into early-death and late-death, with a cut-off of $\mathbf{4 8}$ hours of hospitalization. Among 809 hospitalized cases of COVID-19, 108 patients died (13.35\%) over three months of the study period. The median age of the deceased patients was $60.0(50.0,70.0)$ years; $86.1 \%$ were male. About $85 \%$ had at least one comorbidity with diabetes mellitus $(65.7 \%)$ was the most common one. The most common symptoms were breathlessness $(88.0 \%)$, fever $(65.7 \%)$ and cough $(43.5 \%)$. Nearly $75 \%$ presented with severe disease. Patients had altered biochemical profiles and were treated with different drugs, including antibiotics and steroids. Young age and undernutrition were two characteristic features. Only one-third got intensive care support. The most common cause of death was acute respiratory distress syndrome $(\mathbf{9 5 . 3 7 \%})$. Septic shock and acute myocardial infarction were predominant among early death cases, and uremia, hepatic failure, and hyperglycemic crisis were the predominant causes of late hospital death. The findings of this study will help clinicians and policymakers to take the necessary steps to prevent deaths from COVID-19 in the Bangladeshi population.
\end{abstract}

Keywords: COVID-19, mortality, deceased person, Bangladesh

\section{Introduction}

The coronavirus disease 2019 (COVID-19) is caused by severe acute respiratory syndrome coronavirus 2 (SARS-CoV-2). COVID-19 has been reported for more than 18 crore people, with more than four million deaths worldwide as of 04 July 2021. In Bangladesh, over 14,000 deaths have been reported due to COVID-19. With time, the situation is becoming worse, perhaps because of the new delta variant. The majority of the patients with COVID-19 recover, but only a small portion of the affected persons are at increased risk of fatal outcome ${ }^{1}$. Most of the studies reported several co-morbidities as risk factors of COVID-19 death $^{2}$. However, autopsy reports suggest that COVID-19 is the direct cause of death with little contribution from preexisting health conditions ${ }^{3}$. Septic shock, multi-organ failure, acute and organizing diffuse alveolar damage, pulmonary thromboembolism, etc., are reported as immediate causes of death in COVID-19 cases $^{3,4}$. The characteristics of the deceased person and causes of death may help clinicians identify a population at risk and take appropriate management. However, this aspect of COVID-19 deaths is lacking in South Asian countries, particularly Bangladesh. The aim of this study is to describe the clinical and diagnostic characteristics along with the causes of death in patients with COVID-19.

\section{Materials and Methods}

This retrospective cross-sectional study was conducted among 108 deceased patients of RT-PCR confirmed COVID-19 who had been admitted to Kurmitola General Hospital (KGH), Dhaka during the period of 25 March 2020 to 24 June 2020. Data were extracted from hospital records using a relevant questionnaire. All investigation findings done in the $1^{\text {st }}$ time were included. All information was double-checked before analysis to ensure quality. The severity of COVID-19 was described by WHO interim guidance and the period of death was divided into early and late by cut-off of 48 hours of hospitalization ${ }^{5,6}$. The institutional ethical review board of the Biomedical Research Foundation, Bangladesh, approved the study protocol (Ref. no: BRF/ERB/ 2020/003). Data were expressed in mean \pm standard deviation (SD) or median (interquartile range, IQR) or number (percentages, \%). Analysis was done among available data for each variable using SPSS software version 22.0.

\section{Results}

Among 809 hospitalized cases of COVID-19, 108 patients died $(13.35 \%)$ over three months of the study period. The baseline characteristics of deceased patients with COVID-19 are shown 
in Table 1. The median age of the study population was 60.0 $(50.0,70.0)$ years (min-max: $30-99$ years), with $49 \%$ of the patients being above 60 years. Most of the patients were male $(86.1 \%)$ and had a residence in the urban area $(80.6 \%)$. Only $15.7 \%$ of patients had no comorbidity, while around $60 \%$ had at least two comorbidities. The most common comorbidities were diabetes mellitus (DM) (65.7\%) followed by hypertension, chronic kidney disease (CKD), ischemic heart disease (IHD), etc. More than half of the patients died within four days of hospitalization.

Table 1. Baseline characteristics of deceased COVID-19 patients $(N=108)$

\begin{tabular}{|c|c|}
\hline Variables & Study population \\
\hline Number & 108 \\
\hline Age (years), median (IQR) & $60.0(50.0,70.0)$ \\
\hline \multicolumn{2}{|l|}{ Age groups, no. (\%) } \\
\hline$\leq 40$ years & $11(10.2)$ \\
\hline $41-60$ years & $44(40.7)$ \\
\hline $61-80$ years & $44(40.7)$ \\
\hline e"80 years & $9(8.3)$ \\
\hline \multicolumn{2}{|l|}{ Sex, no. $(\%)$} \\
\hline Male & $93(86.1)$ \\
\hline Female & $15(13.9)$ \\
\hline \multicolumn{2}{|l|}{ Residence, no. (\%) } \\
\hline Urban & $87(80.6)$ \\
\hline Rural & $21(19.4)$ \\
\hline Blood group, no. (\%) & {$[26]^{*}$} \\
\hline A & $11(42.3)$ \\
\hline B & $9(34.6)$ \\
\hline $\mathrm{O}$ & $5(19.2)$ \\
\hline $\mathrm{AB}$ & $1(3.8)$ \\
\hline \multicolumn{2}{|c|}{ Number of comorbidities, no. (\%) } \\
\hline 0 & $17(15.7)$ \\
\hline 1 & $29(26.9)$ \\
\hline e"2 & $62(57.4)$ \\
\hline \multicolumn{2}{|l|}{ Comorbidities, no. (\%) } \\
\hline DM & $71(65.7)$ \\
\hline Hypertension & $51(47.2)$ \\
\hline CKD & $31(28.7)$ \\
\hline IHD & $21(19.4)$ \\
\hline Bronchial asthma & $10(9.3)$ \\
\hline CVD & $5(4.6)$ \\
\hline CLD & $3(2.8)$ \\
\hline COPD & $2(1.9)$ \\
\hline Malignancy & $2(1.9)$ \\
\hline Hospital stay, days & $5.0(2.0,8.0)$ \\
\hline $0-4$ & $56(51.9)$ \\
\hline $5-9$ & $33(30.6)$ \\
\hline$\geq 10$ & $19(17.6)$ \\
\hline
\end{tabular}

Pregnant (1), hypothyroid (3), BEP (1), Parkinson's disease (2), etc.; *[available number]
DM (diabetes mellitus), CKD (chronic kidney disease), IHD (ischemic heart disease, CVD (cerebrovascular disease), CLD (chronic liver disease), COPD (chronic obstructive pulmonary disease)

The average time of onset of symptoms to hospital admission was more than eight days $(8.17 \pm 3.08)$. The most common presenting complaints were breathlessness $(88 \%)$, followed by fever $(65.7 \%)$ and cough (43.5\%). The other clinical symptoms were vomiting, runny nose, bleeding, altered consciousness, anorexia, sore throat, chest pain, abdominal pain, fatigue, etc. Four patients died without any documented symptoms. While tachycardia was present in $57.4 \%$, pyrexia was present in only one patient at presentation. Oxygen saturation was critically low in $70.1 \%$ to $74.4 \%$ of the hospitalized cases were at severe and critical status (Table 2).

Table 2. Clinical features of deceased patients with COVID-19 at presentation $(N=108)$

\begin{tabular}{lc}
\hline Variables & Study population \\
\hline Symptoms at presentation & \\
Onset to hospitalization (days), mean \pm SD & $8.17 \pm 3.08$ \\
Asymptomatic, no (\%) & $4(3.7)$ \\
Difficulty in breathing, no (\%) & $95(88.0)$ \\
Fever, no (\%) & $71(65.7)$ \\
Cough, no (\%) & $47(43.5)$ \\
Signs at presentation & \\
Pulse (beats/ minute), mean $\pm \mathrm{SD}$ & $104.23 \pm 23.40$ \\
Tachycardia (pulse $>100$ beats/minute) & $62(57.4)$ \\
Temperature $\left({ }^{\circ} \mathrm{C}\right)$, mean $\pm \mathrm{SD}$ & $36.0 \pm 0.64$ \\
Pyrexia (Temperature $\left.>38^{\circ} \mathrm{C}\right)$ & $01(0.9 \%)$ \\
Oxygen saturation $(\%)$, mean $\pm \mathrm{SD}$ & $79.19 \pm 16.03$ \\
Low oxygen saturation $(<90 \%)$ & $76(70.4)$ \\
Severity of disease at presentation & \\
Nonsevere (mild to moderate), no (\%) & $28(25.9)$ \\
Severe (severe to critical), no $(\%)$ & $80(74.1)$ \\
\hline
\end{tabular}

Other symptoms: Vomiting (4), runny nose (4), bleeding (3), altered consciousness (3), anorexia (2), sore throat (2), chest pain (2), abdominal pain (1), fatigue (1), etc.

Among 49 available complete blood count reports, more than half had anemia. While leukocytosis was present in $77.6 \%$, both lymphopenia and thrombocytopenia were present in $22.4 \%$ of patients. High neutrophils/lymphocytes ratio and platelets/ lymphocytes ratio were present in $73.5 \%$ and $36.7 \%$ of patients respectively. Among 74 available electrolyte reports, nearly $50 \%$ of patients had hyponatremia. Hyperkalemia $(27 \%)$ was more frequent than hypokalemia $(10.8 \%)$. The mean/median values of all the biochemical variables were abnormal than their respective reference range. A significant percentages of the study population had different abnormal values (Table 3 ).

While different types of antibiotics, both in oral $(n=75)$ and parenteral $(n=69)$ routes, were prescribed in two-thirds of patients, 
Table 3. Investigation findings in deceased patients with COVID-19

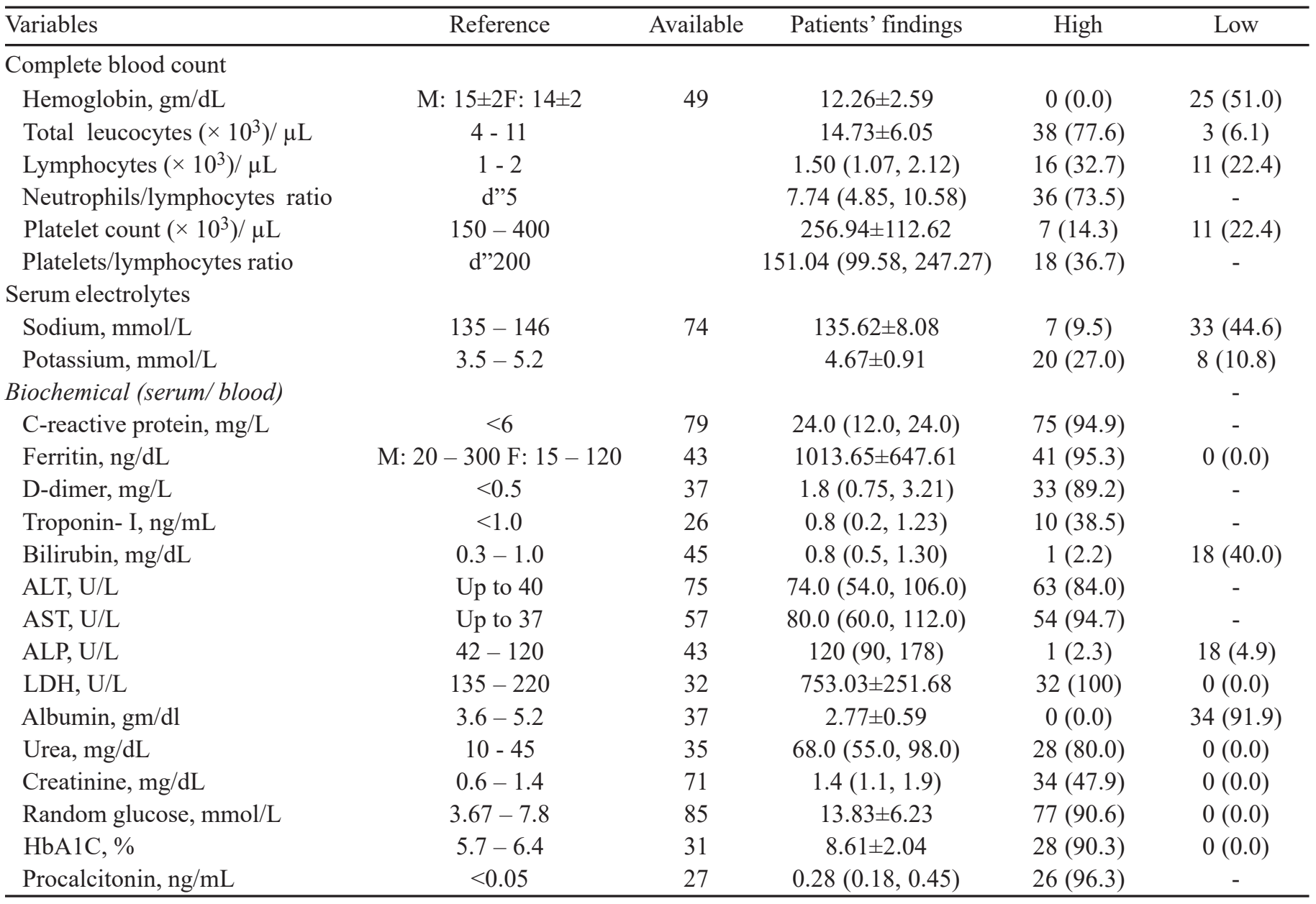

ALT (alanine aminotransferase); AST (aspertate aminotransferase); ALP (alkaline phosphatase); LDH (lactate dehydrogenase)

Data were expressed in mean \pm SD or median (IQR) or frequency (\%)

antivirals were used in one-third $(\mathrm{n}=32)$ of cases. A few cases were treated with ivermectin $(n=8)$, hydroxychloroquine $(n=5)$, tocilizumab $(\mathrm{n}=10)$, and convalescent plasma therapy. Around
$60 \%$ of patients received different types of steroids. Insulin was more prescribed than oral antidiabetic agents (Table 4).

Table 4. Treatment and cause of death of deceased COVID-19 patients

\begin{tabular}{lcl}
\hline Variables & Number (\%) & Details (number) \\
\hline Antibiotics & $75(69.4)$ & Azithromycin (41), coamoxiclav (29), doxicycline (24), others (15) \\
$\quad$ Oral & $69(63.9)$ & Meropenem (59), moxifloxacin (36), ceftriaxone (20), others (16) \\
$\quad$ Intravenous & $32(29.6)$ & Remdesivir (21), favipiravir (10) \\
Antivirals & $8(7.4)$ & \\
Ivermectin & $5(4.6)$ & All enoxaparin subcutaneous \\
Hydroxychloroquine & $75(69.4)$ & Oral: MP (15), Dexa (1); intravenous: MP (19), Dexa (42), HyC (5) \\
Anticoagulants & $64(59.3)$ & \\
Steroids & $8(7.4)$ & All losartan potassium \\
Convalescent plasma therapy & $10(9.3)$ & Metformin (3), glimepiride (2), DPP4 inhibitors (4) \\
Tocilizumab & $14(13.0)$ & Regular (38), glargine (16), conventional premix (3) \\
ACEI/ARB & $7(6.5)$ & \\
Oral antidiabetic agents & $40(37.0)$ & \\
Insulin & $37(34.3)$ & \\
ICU available & &
\end{tabular}

MP (methylprednisolone); Dexa (dexamethasone); HyC (hydrocortisone); DPP4 (dipeptidyl peptidase- 4) 
Figure 1 shows the causes of deaths in patients with COVID-19 according to the duration of hospital stay (cut-off of 48 hours). The most common cause of death was acute respiratory distress syndrome (ARDS) (95.3\%) which occurred almost at the same rate in the early and late-period of hospitalization. While septic shock and acute myocardial infarction were the predominant causes of early death, uremia was more common among patients with late deaths. Hepatic failure, hyperglycemic crisis and hemorrhage were exclusively late causes of death. Electrolyte imbalance was another cause of death which affected both the hospital periods.

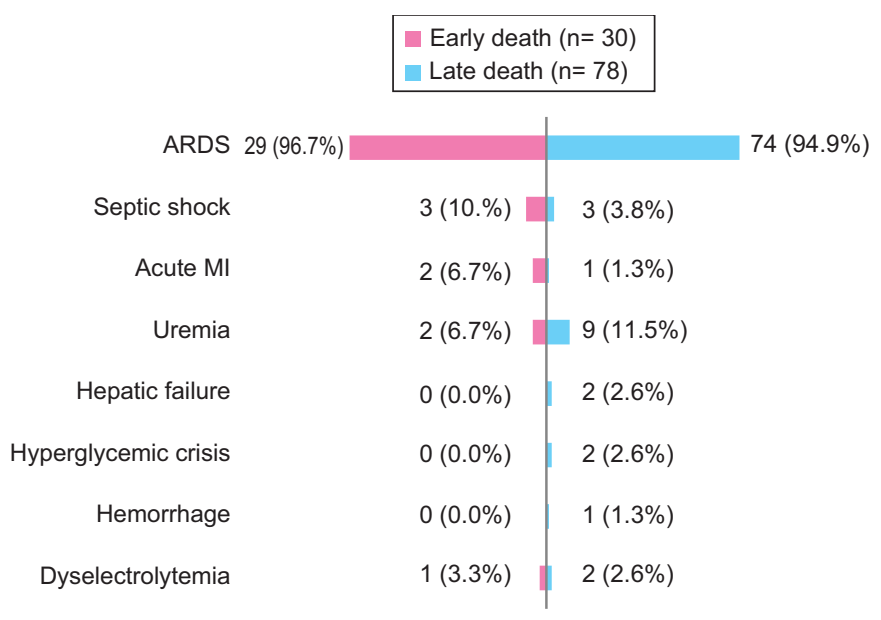

Fig. 1. Causes of death with the duration of admission (cut-off of 48 hours).

\section{Discussion}

This study has revealed a death rate of $13.3 \%$ during the first three months from the initiation of hospitalization of COVID-19 patients. Our finding is consistent with studies reported from other Asian countries ${ }^{2}$. Age e" 65 years was found as a major risk factor for death in patients with COVID-19 in a systematic review and meta-analysis of observational studies ${ }^{7}$. Interestingly, more than half of our study population were younger than 60 years. This finding is in concordance with recent reviews from Bangladesh and India ${ }^{8,9}$; suggests that the population at risk for COVID-19 mortality may differ from other regions of the world, possibly due to genetic or environmental factors or possibly a shorter life expectancy. Other demographic characteristics, clinical features, co-morbidities and duration of hospital stay were similar to other studies $^{10-12}$.

More than half of the study population had anemia with low albumin levels indicating undernutrition as a probable contributing factor of death in COVID-19 patients ${ }^{13}$. Previous studies highlighted obesity; however, the scenario may be different in a developing country like Bangladesh which faces the double burden of malnutrition ${ }^{14}$. However, data regarding body mass index was not documented in hospital records in our study.
More than $90 \%$ of deceased patients had high neutrophils/ lymphocytes ratio, platelets/lymphocytes ratio, C-reactive protein, ferritin, and procalcitonin indicating a severe inflammatory response. Elevated procalcitonin also indicates bacterial coinfection or probably a direct consequence of viral sepsis ${ }^{15}$. Similarly, a significant proportion of patients with high D dimer, suggesting a prothrombotic state in COVID-19 ${ }^{16-18}$. High liver enzymes and creatinine were probably systemic responses of COVID-19 infection and may affect prognosis ${ }^{19}$. High random glucose in around $90 \%$ of patients was due to inflammatory response and steroid use. High $\mathrm{HbA}_{1 \mathrm{C}}(\%$, found among patients with diabetes mellitus also indicates poor glycemic control, an important risk factor for poor outcome ${ }^{20}$.

Several treatment options were tried initially without much evidence. However, most patients could not manage ICU due to unavailability in a 10-bedded ICU in the study hospital. We found ARDS as the main cause of death in patients with COVID-19 rather than associated co-morbidities. Slater et al. (2020) also showed death in COVID-19 as a direct consequence of the virus, similar to an autopsy report of 26 deceased COVID-19 patients $^{4,21}$.

There are several limitations of our study. The sample size is small. All the data are retrospective and secondary, with a possibility of under-reporting along with many incomplete data.

\section{Conclusions}

Our study findings showed several characteristics and unique features (young age, undernutrition) of deceased patients with COVID-19 than other parts of the world. These findings would help clinicians and policymakers tailor management strategies, facilitate decision-making, and ultimately improve patient outcomes.

\section{Declarations}

\section{Acknowledgments}

We are grateful to Brigadier General Jamil Ahmad, director, Kurmitola General Hospital (KGH); Md. Ashek Ali, statistical officer, $\mathrm{KGH}$, for their generous support in data collection.

\section{Competing interest}

None of the authors has any conflict of interest to declare

\section{Ethics approval and consent to participate and publish}

The institutional review board of the Biomedical Research Foundation, Bangladesh, approved the study protocol (Ref. no: BRF/ERB/2020/003).

\section{Funding}

Not applicable

\section{References}

1. Worldometer. COVID live update. Accessed on $4^{\text {th }}$ June, 2021. URL: https://www.worldometers.info/coronavirus/. 
2. Noor FM and Islam MM. Prevalence and associated risk factors of mortality among COVID-19 patients: A Meta-Analysis. J Community Health. 2020;45(6):1270-1282. doi:10.1007/s10900-020-00920-x.

3. Schaller T, Hirschbühl K, Burkhardt K, Braun G, Trepel M, Märkl B and Claus R. Postmortem examination of patients with COVID19. JAMA. 2020;323(24): 2518-2520. doi:10.1001/jama.2020.8907.

4. Elezkurtaj S, Greuel S, Ihlow J, Michaelis EG, Bischoff P, Kunze CA, et al. 2021. Causes of death and comorbidities in hospitalized patients with COVID-19. Sci Rep. 11:4263. https://doi.org/10.1038/s41598-02182862-5.

5. World Health Organization 2020. Clinical management of COVID-19: interim guidance, 27 May 2020. Accessed: August 2, 2020. Available at: https://apps.who.int/iris/handle/10665/332196.

6. Garcia-Vidal C, Fernández-Sabé N, Carratalà J, Díaz V, Verdaguer R, Dorca J, Manresa F and Gudiol F. Early mortality in patients with community-acquired pneumonia: causes and risk factors. Eur Respir J. 2008;32(3):733-739. doi: 10.1183/09031936.00128107.

7. Parohan M, Yaghoubi S, Seraji A, Javanbakht MH, Sarraf P and Djalali M. Risk factors for mortality in patients with Coronavirus disease 2019 (COVID-19) infection: a systematic review and meta-analysis of observational studies. Aging Male. 2020;23(5):1416-1424. doi: 10.1080/ 13685538.2020 .1774748 .

8. Siam MH, Hasan MM, Tashrif SM, Khan MH, Raheem E and Hossain MS. Insights into the first seven-months of COVID-19 pandemic in Bangladesh: lessons learned from a high-risk country. Heliyon. 2021;7(6):e07385.https://doi.org/10.1016/j.heliyon.2021.e07385.

9. Upadhyay AK and Shukla S. Correlation study to identify the factors affecting COVID-19 case fatality rates in India. Diabetes Metab Syndr. 2021;15(3):993-999. https://doi.org/10.1016/j.dsx.2021.04.025.

10. Jalili M, Payandemehr P, Saghaei A, Sari HN, Safikhani H and Kolivand P. Characteristics and mortality of hospitalized patients with COVID-19 in Iran: a national retrospective cohort study. Ann Intern Med. 2021;174(1):125-127. doi: 10.7326/M20-2911.

11. Wortham JM, Lee JT, Althomsons S, Latash J, Davidson A, Guerra K, et al. Characteristics of persons who died with COVID-19 - United States, February 12-May 18, 2020. MMWR Morb Mortal Wkly Rep.
2020;69(28):923-929. doi:10.15585/mmwr.mm6928e1.

12. Berenguer J, Ryan P, Rodríguez-Baño J, Jarrín I, Carratalà J, Pachón J, et al. Characteristics and predictors of death among 4035 consecutively hospitalized patients with COVID-19 in Spain. Clin Microbiol Infect. 2020;26(11):1525-1536. doi:10.1016/j.cmi.2020.07.024.

13. Wu MA, Fossali T, Pandolfi L, Carsana L, Ottolina D, Frangipane V, et al. Hypoalbuminemia in COVID-19: assessing the hypothesis for underlying pulmonary capillary leakage. J Intern Med. 2021;289(6):861872. doi: 10.1111 /joim. 13208 .

14. Mertens E and Peñalvo JL. The burden of malnutrition and fatal COVID19: a global burden of disease analysis. Front Nutr. 2021;7:619850. doi:10.3389/fnut.2020.619850.

15. Li H, Liu L, Zhang D, Xu J, Dai H, Tang N, et al. SARS-CoV-2 and viral sepsis: observations and hypotheses. Lancet. 2020;395(10235):15171520. doi: 10.1016/S0140-6736(20)30920-X.

16. Zhou F, Yu T, Du R, Fan G, Liu Y, Liu Z, et al. Clinical course and risk factors for mortality of adult inpatients with COVID-19 in Wuhan, China: a retrospective cohort study. Lancet. 2020;395(10229):1054-1062. doi:10.1016/S0140-6736(20)30566-3.

17. Zhang B, Zhou X, Qiu Y, Song Y, Feng F, Feng J, et al. Clinical characteristics of 82 cases of death from COVID-19. PLoS One. 2020;15(7):e0235458. doi:10.1371/journal.pone.0235458.

18. Lopez-Castaneda S, García-Larragoiti N, Cano-Mendez A, Blancas-Ayala K, Damian-Vázquez G, Perez-Medina AI, et al. Inflammatory and prothrombotic biomarkers associated with the severity of COVID-19 infection. Clin Appl Thromb Hemost. 2021;27:1076029621999099. doi:10.1177/1076029621999099.

19. Tian W, Jiang W, Yao J, Nicholson CJ, Li RH, Sigurslid HH, et al. Predictors of mortality in hospitalized COVID-19 patients: a systematic review and meta-analysis. $J$ Med Virol. 2020;92(10):1875-1883. doi:10.1002/jmv.26050.

20. Chen L, Sun W, Liu Y, Zhang L, Lv Y, Wang Q, et al. 2021. Association of early-phase in-hospital glycemic fluctuation with mortality in adult patients with coronavirus disease. Diabetes Care. 2019;44(4):865-873. doi: $10.2337 / \mathrm{dc} 20-0780$.

21. Slater TA, Straw S, Drozd M, Kamalathasan S, Cowley A and Witte KK. Dying 'due to' or 'with' COVID-19: a cause of death analysis in hospitalised patients. Clin Med. 2020;20(5):2p. DOI: https://doi.org/ 10.7861/clinmed.2020-0440. 